\title{
Trauma care in Africa: A status report from Botswana, guided by WHO’s “Guidelines for Essential Trauma Care”
}

Terje Peder Hanche-Olsen ${ }^{12}$ Lulseged Alemu ${ }^{3}$ Asgaut Viste ${ }^{45}$ Torben Wisborg ${ }^{67}$ Kari S Hansen ${ }^{68}$

${ }^{1}$ Unit for International Collaboration, Haukeland University Hospital, Bergen; Norway

2 Department of Anaesthesiology, Oslo University Hospital, Oslo, Norway

${ }^{3}$ Department of Surgery, Nyangabgwe Referral Hospital, Francistown, Botswana

${ }^{4}$ Department of Surgery, Haukeland University Hospital, Bergen, Norway

${ }^{5}$ Department of Surgical Sciences, University of Bergen, Bergen, Norway

${ }^{6}$ The BEST Foundation: Better \& Systematic Team Training, Hammerfest Hospital, Norway

${ }^{7}$ Anaesthesia and Critical Care Research Group, Faculty of Health Sciences, University of Tromsø, Tromsø, Norway

${ }^{8}$ Longyearbyen Hospital, Longyearbyen, Norway

\section{Corresponding author:}

Terje Peder Hanche-Olsen

Oslo University Hospital

Department of Anaesthesiology

Kirkeveien 166 
0407 Oslo

\section{NORWAY}

e-mail: hancheolsen@hotmail.com

Phone + 4795124814 or +4722119690

\section{Funding}

The Laerdal Foundation for Acute Medicine financially supported this project

\section{Conflicts of interest}

None

\section{Acknowledgements}

Dr Celestine Mbangtang and the members of the trauma committee at Nyangabgwe Hospital provided invaluable assistance in initiating the project and during the project period. Dr Thapelo Montshiwa and Mma Malatsi from Princess Marina Hospital were of utmost importance in all parts of the project. Thanks also to Dr Chiapo Lesetedi, Dr Mompati Mmalane and Dr Kolaatamo Malefho from the Ministry of Health, Botswana, for financial and logistical support. 


\begin{abstract}
Background Trauma represents a significant and increasing challenge to health care systems all over the world. This study aimed to evaluate the trauma care capabilities of Botswana, a middle-income African country, by applying the World Health Organization's Guidelines for Essential Trauma Care.
\end{abstract}

Methods All 27 government (16 primary, nine district, and two referral) hospitals were surveyed. A questionnaire and checklist, based on "Guidelines for Essential Trauma Care” and locally adapted, were developed as situation analysis tools. The questionnaire assessed local trauma organization, capacity, and the presence of quality improvement activity. The checklist assessed physical availability of equipment and timely availability of traumarelated skills. Information was collected by interviews with hospital administrators, key personnel within trauma care, and through an on-site physical inspection.

Results Hospitals in Botswana are reasonably well supplied with human and physical resources for trauma care. Deficiencies were noted. At the primary and district levels, both capacity and equipment for airway/breathing management and vascular access was limited.

Trauma administrative functions were largely absent at all levels. No hospital in Botswana had any plans for trauma education, separate from or incorporated into other improvement activities. Team organization was non-existent and training activities in the emergency room very limited. 
Conclusions This study draws a picture of trauma care capabilities of an entire African country. Despite good organizational structures, Botswana has room for substantial improvement. Administrative functions, training, human and physical resources could be improved. By applying the guidelines, this study creates an objective foundation for improved trauma care in Botswana. 


\section{Introduction}

Mortality and morbidity owing to trauma is one of the leading health problems in the world today. Worldwide, an estimated nearly 6 million people die annually due to injuries [1].

One in ten deaths globally is caused by trauma, and nine in ten occur in a low- or middleincome country (LMIC) [2].

Improvements in the organization of trauma care results in reduced trauma-related mortality. Nathens et al. reported a 9\% lower injury mortality rate in the United States (US) with a regional trauma system [3], and an $8 \%$ reduction in mortality owing to motor vehicle accidents (MVA) after trauma system implementation [4]. In a systematic review of trauma literature from the US, Mann et al. found that implementation of systematic trauma care reduces mortality [5]. In a low-income setting from northern Iraq and Cambodia, Husum et al. found a significant impact on trauma outcome after implementation of a prehospital trauma system [6].

Survival after injuries varies considerably between countries of different economic levels. Mock et al. found increased mortality with decreasing economic level in a study comparing outcomes for seriously injured patients in Kumasi, Ghana, Monterey, Mexico, and Seattle, US, and that the majority of deaths occured before arrival at a hospital, concluding that improvement efforts in LMIC countries should focus on injury prevention, prehospital treatment, and emergency room care [7].

The World Health Organization (WHO) developed the "Guidelines for Essential Trauma Care” (EsTC) in collaboration with the International Association for the Surgery of Trauma and Surgical Intensive Care. The guidelines were released in 2004 to "provide recommendations for improvements in trauma care through better planning and 
organization adjusted to economic realities in a low or middle income setting” [8]. The guidelines were previously used for a situation analysis in Ghana, Vietnam, Mexico, India [9], Equador [10], Morocco [11], Cambodia [12] and to revise national trauma guidelines in South Africa [13].

In 2005, a Norwegian-Botswana government to government project called ”Human Resources to Assist Ministry of Health, Botswana” was established. Trauma care was identified as an area for collaboration. As part of and prior to a trauma training program, we wanted to describe and analyze the trauma system components in Botswana based on recommendations made by WHO’s “Guidelines for Essential Trauma Care” (EsTC) in order to highlight areas of improvement, and to establish a baseline against which development can be compared. This is, to our knowledge, the first time this document has been used for a situation analysis in an entire country.

\section{Methods and setting}

Study settings

Botswana is an upper middle-income country in Southern Africa with a gross national income per capita of \$ 6,260 (2009) [14]. The country has had one of the fastest growing economies in the world since its independence in 1966.

The population is estimated at 1.95 million, and $60 \%$ live in urban areas [15].

Communicable diseases like HIV/AIDS and tuberculosis account for 83\%, non communicable diseases account for $10 \%$ and injuries for $7 \%$ of years of potential life lost. [16]. Twenty thousand were registered as injured and 500 were killed after MVA in 2008 [17], giving a mortality rate of 25 per 100,000 which is above the worldwide middle 
income average of 19.5 and far above the high income European region average of 7.9 [2].

Other significant contributors to the burden of injury in Botswana are falls and domestic violence.

Health care in Botswana

Botswana has a well organized health care system. Primary health care is organized at different levels, from mobile stopping points through health posts to clinics. Clinics are staffed by nurses and occasionally also a doctor, and serve a population of up to a few thousand [18]. The hospital system is divided into three levels [19].

Primary hospitals have few doctors and provide basic in- and outpatient services. These hospitals are non-specialist facilities with laboratory and radiological services. Some have basic surgical capabilities such as those for caesarean section and ectopic pregnancies. Emergencies are usually received in an examination room in the outpatient department (OPD). Typically, they have a catchment area of 10,000-30,000 inhabitants.

District hospitals are the first level for referral. Most are non-specialist facilities, but a few provide specialist services like gynaecology, paediatrics, surgery, otolaryngology, and anaesthesiology. They have a catchment area of up to 250,000 inhabitants. Most have a more or less developed emergency room (treatment room for all emergencies) in the admission area of the hospital. They have operating theatres and anaesthesia service is provided by nurse anaesthetists. Some hospitals use their regular outpatient department rooms for emergencies. These rooms are often small and not equipped for emergency care.

The two referral hospitals, Nyangabgwe in the north and Princess Marina in the south, provide specialist services in most fields. Both are regional trauma centres, with system responsibility in their region and surgical subspecialties. Injured persons are 
received in an emergency room in the Accident \& Emergency (A\&E) Department by nonspecialist doctors and nurses.

Government ambulances are generally in good condition, but are sparsely or not equipped. Large hospitals have 5-7 ambulances, small 3-5 and clinics may have one. The two-person crew has no medical training. Trauma victims are often transported to hospital or nearest clinic by police or private cars. A private emergency medical transportation system is accessible through payment or a medical aid system. There is no national emergency number.

Trauma team training program

As part of the bilateral cooperation between Botswana and Norway, in 2007 it was agreed to providing a trauma training program to all Botswana government hospitals. The training concepts were developed in Norway, and are known as the Better and Systematic Team Training (BEST) approach [20], a non profit training concept with a main focus on multiprofessional trauma team training using simulations. The training programme period was from November 2007 until November 2009.

The situation analysis of the trauma care capabilities in the country was done prior to training at the individual facilities.

Data collection

A total of 27 facilities at three different levels were surveyed, covering all somatic government hospitals. Four private hospitals, three of which were mining hospitals, and clinics, were not a part of this study. Data for physical resources and skills for one district 
hospital were missing. For data collection, we developed two forms; a questionnaire and a checklist.

A draft for a checklist and a questionnaire based on WHO: "Guidelines for Essential Trauma Care” (EsTC) were developed after the pilot training course in 2007 by the first author, who at that time was employed by Ministry of Health, Botswana, in collaboration with Norwegian training partners. This draft was revised according to WHO: "Checklist for surveys of trauma care capabilities" and input from the trauma committee in Francistown and other local partners.

The checklist assessed 64 items of equipment, whether it was immediately available in the emergency room, available in the hospital, or not available at all for the three hospital levels. Equipment with similar characteristics were for the purpose of the paper grouped together; for example oxygen wall/oxygen bottle/oxygen concentrator were grouped as oxygen supply. Items assessed, but of less importance for immediate hospital based trauma care and diagnosis, were left out from the paper (for example otoscope, Magills forceps), making the number of items to 34 . An item was considered available if it could be presented in working condition or for items of multiple sizes that most sizes of the item were available (oropharyngeal airways, endotracheal tubes). Desirable and possibly required items were upgraded to essential because of Botswana status as middle income country, according to the classification and recommendation of the EsTC checklist. Next the checklist assessed timely availability of 59 trauma-related skills separated into $24 \mathrm{~h}$ per day/7 days per week/365 days per year (24/7/365); sometimes; and never. The 24/7/365 availability is separate from "sometimes" by being a service that can be provided at all times, even during the night, and on weekends and holidays. Items and procedures related 
to drugs and rehabilitation were excluded. Thirty four skills considered most relevant and important according to established principles for acute hospital based trauma care, were selected for the paper (example; airway, breathing and circulation were considered most important, but capabilities for fracture treatment is also relevant for prevention of disability). The first 3 elements of the checklist (airway, breathing, circulation) are shown in Appendix 1.

The questionnaire assessed local trauma resources in terms of manpower, organization and presence of quality improvement activity and is shown in Appendix 2. The terms used in Appendix 2 are defined in Appendix 3.

Norwegian team members interviewed local personnel within trauma care using the questionnaire and the checklist prior to the first course at each hospital. In primary and district hospitals these persons were the head or acting head of the facility, the doctor and nurse responsible for the OPD, a radiographer and a laboratory technician. In larger facilities it would also include the head of the accident and emergency and surgical departments. All interviews were performed in a person-to person setting. Next, at least one of the Norwegian team members inspected the facility together with the previously mentioned local personnel who presented their facility and the different departments. The interviews and data collection at the individual hospital were done by the same person. An important part of the inspection was the evaluation of the emergency room (OPD/A\&E) during the simulations/team training part of the training programme, which provided a good basis for an objective assessment of local trauma care capacity/function. The final scoring or assessment for the checklist and the questionnaire resulted from synthesis of information gained through interviews, physical inspection, simulation part of the program 
and finally discussion within the Norwegian team. The training programme and thereby the collection of data went on for two years (November 2007 to November 2009). In total, for all hospitals, three persons were responsible for data collection, after careful discussion of definitions and assessment criteria to ensure similarity in observations.

The study was approved by the Research and Development Office, Ministry of Health, Botswana.

\section{Results}

Hospital characteristics and human resources for trauma care in Botswana are summarized in Table 1. In primary hospitals there were no specialists in surgery, four had no and 10 had one nurse anaesthetist.

No hospitals had local trauma guidelines, systems for audit, education plans, defined trauma teams, or conducted educational trauma simulations. In the two referral hospitals inservice lectures were conducted aiming at training A\&E staff about triage and preparedness for mass casualties. Two hospitals had a local trauma committee. The first hospital was the initiator of the BEST training program; the second was established in response to the pilot course. One hospital had a trauma manual. All hospitals registered their admissions and outpatient visits. Traumas were included in these registers in all except four hospitals. Separate trauma registries were found in two primary hospitals, one district hospital, and one referral hospital and contained to varying extent information on sex, age, time of visit/admission, main injury mechanism, main findings, but not detailed information for injury severity scoring. In the referral hospital, injury severity scoring was about to be implemented. 
The physical resources for initial resuscitation and diagnosis in trauma victims at the three hospital levels in Botswana are summarized in Table 2.

Oxygen availability was good at all hospital levels. Oxygen face masks were generally without reservoir. Physical resources were fairly good for basic airway management at the primary and district hospital levels. There were major deficiencies at the primary and district hospital levels regarding physical resources for advanced airway/breathing management; self-inflating ventilation bags, facemasks, laryngoscopes, endotracheal tubes, and surgical/needle cricothyrotomy sets. This especially applied to paediatric equipment. Chest tubes for children were not available in 11 facilities.

Ventilators were present in the emergency room in both referral and one district hospital, for the remaining district hospitals available in main operation theatres (anaesthesia machine). Ventilators were found even at primary level, but are not deemed essential, according to the guidelines.

Vascular access was in general limited to peripheral percutaneous intravenous access. Equipment for intraosseus access was, with three exceptions, not part of the armamentarium at any level. Sets for surgical cut down were found nine places.

There were shortages of monitoring equipment at all levels. Pulse oximeters were found in 22 hospitals, ECG in 15. In six respectively four facilities, these items were found in emergency rooms/OPD rooms used for emergencies and for the rest in main operation theatre. Laparotomy sets were present in 13 primary and all eight district facilities.

Diagnostic capabilities for basic trauma care were present at all hospital levels including plain x-ray films, and even portable x-ray machines in some places. In one hospital, lack of radiological services was due to a technical break down. Basic laboratory 
services, including haemoglobin and electrolytes, were available at all hospitals. Some hospitals reported lack of reagents. All 26 hospitals had blood transfusion capabilities, but limited supplies, both in the type and number available. Ultrasound was, except for two primary facilities, a service of district and referral hospitals and was performed by radiographers.

The knowledge, skills, and human resources were assessed in the format of questioning: "How often will your hospital be able to perform the following procedure?" stratified into "24/7/365", "sometimes", and "never”. The results are summarized in Table 3.

Generally, primary hospitals are basic facilities with a limited capacity for severe trauma care. Except for administration of oxygen, the skills for basic and advanced airway management and spine immobilization were limited, especially at the primary hospital level, but also at the district hospital level. Three hospitals were not able to provide endotracheal intubation at all, for 18 this service could be provided on a "sometimes" basis. Recovery position was an unknown procedure in 11 hospitals. Chest tube insertion was a procedure that could be provided by all doctors caring for injuries in only 11 locations.

The skills for recognition and monitoring of the bleeding patients as defined in the Guidelines were limited at the primary and district level. Five respective six hospitals on these levels were assessed to have sufficient skills. Intraosseus vascular access was with three exceptions an unknown procedure, whereas venous cut down could be provided "sometimes" or "always" in 21 hospitals. Control of external haemorrhage by deep packing was 24-h available in one hospital, "sometimes" in seven, wrapping of potential pelvic fractures 24-h available in one and "sometimes" in six facilities. 
Generally, we found that primary and district hospitals had limited physical and human resources for managing specific injuries. Five hospitals reported skills and equipment for 24-h emergency laparotomy. Eleven hospitals could provide emergency laparotomy “sometimes”. Diagnostic peritoneal lavage was not a commonly performed procedure, in contrast to a "four quadrant abdominal tap."

All except one hospital were familiar with the Glasgow Coma Scale Score and recognition of altered mental status, but no hospital would consider performing a burr hole in a head injury patient developing unilateral pupil dilatation.

Ultrasound could be performed in seven of eight district hospitals, but only in half of them on a $24 \mathrm{~h}$ basis.

Basic orthopaedic services like closed reduction of fractures was performed in all hospitals, even primary ones, but basic immobilization could not be provided in three due to lack of immobilization devices. More advanced orthopaedic interventions were only available at referral hospitals. The same conditions applied to major soft tissue damage repair.

\section{Discussion}

This study establishes the status of trauma care in the middle-income African country of Botswana before introduction of a national trauma team training programme. While similar studies have focused on regions of countries $[9,10,11,21]$ or nationally representative samples from all over the country [12], our study provides for the first time a picture of hospital based trauma care capabilities in an entire country. 
The main findings were that Botswana is insufficiently supplied with human and physical resources for basic trauma care. At the tertiary level, equipment, personnel, and diagnostic capabilities approximate the recommendations from the WHO "Guidelines for Essential Trauma Care”. Tertiary hospitals have the range of specialists needed for basic trauma care and treatment of most specific injuries. Primary and district facilities did not meet the recommendations at several points when it came to physical resources and skills. These findings seem to correlate with findings in similar assessments from South America, Africa and from Asia [9-12, 21]. The highest level facilities assessed seems to be more adequately staffed, equipped and trained, although with deficiencies, while districts are covered by generalist hospitals or clinics with very limited trauma care capacity and substantial trauma load, especially along the major roads, which highlights the need for improved trauma care in rural areas.

Important elements of hospital-based trauma care are human resources (staffing and training), physical resources (infrastructure, equipment and supplies), and administrative functions/organization.

Staffing and training

Trained health care workers are essential for improved outcomes [22]. Studies from other low- and middle-income countries have shown that prehospital trauma training programs can reduce mortality $[6,23,24]$. The same was shown for hospital-based care from Trinidad where regular implementation of an ATLS-course led to reduced mortality in severely injured patients [25]. All primary and the majority of district hospitals in Botswana are managed by general practitioners (GP) with limited surgical training. In order to serve at primary or district hospitals, doctors need surgical experience in order to perform at least caesarean section. If they do not have relevant training, they are required to 
practice at a referral hospital before they start working at a primary or district hospital.

There are no such or other training requirements for trauma care, even if severe traumas are quite common and referral distances are long.

The system of rotations is another important issue. All health care workers are employed by the Ministry of Health, and not employed at the individual facility. Staffs rotate between facilities as well as internally between departments rather frequently, unless they have any kind of speciality, which only applies to a minority. These facts combined with non-existent trauma education and limited training activities make capacity building difficult.

Lack of personnel and particularly specialists is a significant problem in Africa [26]. We found that primary and district hospitals in Botswana had limited human and physical resources and therefore, limited diagnostic capabilities for managing specific injuries. Due to lack of personnel, few hospitals could in practice provide 24/7/365 anaesthesia service, and a nurse anaesthetist was on call from home after hours, indicating that time to definitive care (airway control, haemorrhage control) might be prolonged. Time to relevant intervention is related to outcome [27] and so is timely arrest of internal bleeding [28]. It should therefore be noted that only few hospitals could provide emergency laparotomy on a 24/7/365 basis; therefore, most such patients must be transferred to the next level even though there is no specialist service in the receiving facility. During the daytime, this can be achieved by air lift, but only by road ambulance after dark. As an example, a primary hospital might be located $300 \mathrm{~km}$ away from the district hospital, which is also only occasionally staffed with a surgeon, and $700 \mathrm{~km}$ from a tertiary level hospital, which always has surgical capabilities. In countries without a formal EMS-system, it is known that the majority of trauma deaths occur prehospital, for example in Ghana more than $80 \%$ 
[7], partly due to the fact that transport from accident scene to hospital may take several hours. This is also the case from many locations in Botswana.

Trauma teams with preassigned roles are increasingly implemented in developed countries and have been shown to reduce mortality [29]. A major benefit from trauma team organization is a reduction in time to definitive surgery [30]. Efforts have been made to introduce the concept to African countries. However size and composition may differ from developed countries due to resource restrictions. An evaluation of a trauma team training programme in Tanzania reported significant knowledge gain and improved performance after the course [31].

\section{Equipment}

We found that there were major deficiencies regarding equipment, especially at the primary and district levels. Because some hospitals at the same level had certain items and some did not, the lack of items was at least partly caused by poor organization and not by availability, as has been shown in other studies [9]. In many hospitals, equipment missing in the emergency room was found in another area of the hospital not being utilized optimally, thus the equipment was not available for emergency use. This was also the case for more costly equipment like that for electro-cardiac monitoring and pulse oximeters.

\section{Administrative functions}

Noteworthy was the nearly complete absence of trauma administrative functions, like a trauma committee at national or local level and quality improvement activities defined as "a method of improving care by monitoring the elements of diagnosis, treatment and 
outcome” [32]. Such improvement programs has documented effect even in LMIC in different fields and in trauma in Khon Kaen in Thailand [33], where identification of preventable deaths and corrective interventions led to a reduction in mortality. A key component of quality improvement is a trauma registry with severity scoring for outcome evaluation and monitoring of process of care [32]. However, creation and maintenance of a trauma registry requires substantial investments of both money and human resources [34] which might impose a challenge for LMIC.

\section{Limitations}

The strength of this study was that it was a national study with complete coverage of one country's hospital system. However, the study did not cover clinics, which receive a substantial number of trauma cases from rural areas, or prehospital care. The importance of the prehospital phase of trauma management although not covered in our study, must be underlined. The study was limited to government hospitals. However, private hospitals play only a minor role in the Botswana health care system, and to an even lesser degree in trauma care.

Our study was focused on infrastructure, human and physical resources and did not cover outcome measures. Improved outcome is what improvement efforts are about. However, the basis for improved outcome is necessary infrastructure, equipment and human capacity.

Data collection went on over a two-year period. Theoretically, the status of a specific hospital might have changed during that time period. We do, however, find this unlikely, 
because we did not find any significant differences between hospitals surveyed early versus later during the study period.

Information about trauma care skills depended on information provided by care givers, and is therefore highly subjective. Informants may tend to over report their capabilities. Even our own observations will to some degree be subjective, especially for skills, although we tried our best to make an objective assessment.

\section{Conclusion}

Despite a quite well-developed public health care system, relatively little attention has been paid to injury compared to other major health challenges in Botswana, like HIV/AIDS.

This study has, aided by “Guidelines for Essential Trauma Care”, highlighted the current status of hospital-based trauma care in this country, and indicates several areas for improvement. Improvements in availability and organization of equipment for basic trauma care are probably of major importance for patient care and outcomes, especially in primary and district hospitals.

Trauma administrative functions need to be developed at all levels. Trauma care should be incorporated into each hospital's training and quality improvement activities. Attention should be given to initial assessment, team organization, and team work. All doctors caring for injuries must be trained in life saving procedures according to hospital level and national policies. 


\section{References}

1. WHO (2004) The Global burden of disease: 2004 update. World Health Organization, Geneva

2. WHO (2009) Global status report on road safety. World Health Organization, Geneva

3. Nathens AB, Jurkovich GJ, Rivara FP, et al (2000) Effectiveness of state trauma systems in reducing injury-related mortality: a national evaluation. J.Trauma 48:2530

4. Nathens AB, Jurkovich GJ, Cummings P, et al (2000) The effect of organized systems of trauma care on motor vehicle crash mortality. JAMA 283:1990-1994

5. Mann NC, Mullins RJ, MacKenzie EJ, et al (1999) Systematic review of published evidence regarding trauma system effectiveness. J.Trauma 47: S25-S33

6. Husum H, Gilbert M, Wisborg T, et al (2003) Rural prehospital trauma systems improve trauma outcome in low-income countries: a prospective study from North Iraq and Cambodia. J.Trauma 54:1188-1196 
7. Mock CN, Jurkovich GJ, nii-Amon-Kotei D, et al (1998) Trauma mortality patterns in three nations at different economic levels: implications for global trauma system development. J.Trauma 44:804-812

8. Mock C, Lormand JD, Goosen J, et al (2004) Guidelines for essential trauma care. World Health Organization, Geneva

9. Mock C, Nguyen S, Quansah R, et al (2006) Evaluation of Trauma Care capabilities in four countries using the WHO-IATSIC Guidelines for Essential Trauma Care. World Journal of Surgery 30:946-956

10. Aboutanos MB, Mora F, Rodas E, et al (2010) Ratification of IATSIC/WHO's guidelines for essential trauma care assessment in the South American Region. World J.Surg 34:2735-2744

11. N Tacfouti, J.A. Bhatti, C Nejjari, et al (2010) Emergency trauma care for severe injuries in a Moroccan Region: Conformance to French and World Health Organization standards. J for Healthcare Quality 33:30-38

12. Nakahara S, Saint S, Sann S, et al (2009) Evaluation of trauma care resources in Health Centers and Referral Hospitals in Cambodia. World J Surg 33:874-885

13. Hardcastle TS, Steyn E, Boffard K, et al (2011) Guideline for the assessment of trauma centres for South Africa. S Afr Med J 101:189-194

14. World Development Indicators \& Global Development Finance 2010. World Bank Data, The World Bank, Washington, USA. Available on the internet at www.databank.worldbank.org 
15. The World Bank (2010) World Development Indicators \& Global Development Finance 2009. World Bank Data., The World Bank, Washington, USA. Available on the internet at www.databank.worldbank.org

16. WHO (2006) World Health Statistics. World Health Organization, Geneva

17. MVA (2008) Annual Report 2008. Motor Vehicle Accident Fund, Botswana

18. Seloilwe ES, Thupayagale-Tshweneagae G (2007) Community mental health care in Botswana: approaches and opportunities. Int. Nurs. Rev 54:173-178

19. WHO (2009) Country Cooperation Strategy, 2008-2013 Botswana. World Health Organization Regional Office for Africa

20. Wisborg T, Brattebo G, Brinchmann-Hansen A, et al (2008) Effects of nationwide training of multiprofessional trauma teams in norwegian hospitals. J.Trauma 64:1613-1618

21. Arreola-Risa C, Mock C, Vega RF, et al (2006) Evaluating trauma care capabilities in Mexico with the World Health Organization's Guidelines for Essential Trauma Care publication. Rev.Panam.Salud Publica 19:94-103

22. Mock C, Kobusingye O, Joshipura M, et al (2005) Strengthening trauma and critical care globally. Curr.Opin.Crit Care 11:568-575.

23. Ali J, Adam RU, Gana TJ, et al (1997) Trauma patient outcome after the Prehospital Trauma Life Support program. J.Trauma 42:1018-1021.

24. Arreola-Risa C, Mock CN, Lojero-Wheatly L, et al (2000) Low-cost improvements in prehospital trauma care in a Latin American city. J.Trauma 48:119-124. 
25. Ali J, Adam R, Butler AK, et al (1993) Trauma outcome improves following the advanced trauma life support program in a developing country. J.Trauma 34:890898.

26. WHO (2006) World Health Report 2006 - Working Together for Health. World Health Organization, Geneva

27. Driscoll PA, Vincent CA (1992) Variation in trauma resuscitation and its effect on patient outcome. Injury 23:111-15

28. Henderson KIM, Coats TJ, Hassan TB (2000) Audit of time to emergency trauma laparotomy. British J of Surgery 87:472-76

29. Petrie D, Lane P, Stewart TC (1996) An evaluation of patient outcomes comparing trauma teams activated versus trauma teams not activated using TRISS analysis. J Trauma 41:870-875

30. Driscoll, PA, Vincent CA (1992) Organizing an efficient trauma team. Injury 23:10710.

31. Bergman S, Deckelbaum D, Lett R, et al (2008) Assessing the impact of the trauma team training program in Tanzania. J.Trauma 65:879-883

32. WHO (2009) Guidelines for quality improvement programmes. World Health Organization, Geneva

33. WHO (2010) Strengthening the care for the injured: Success stories and lessons learned from around the world. World Health Organization, Geneva 
34. Moore L Clark.DE (2008) The value of trauma registries. Injury 39:686-95. 
Table 1.

Hospital characteristics and human resources for trauma care in Botswana.

Number Primary Hospital District hospital

Referral Hospital
$n=16$
$\mathrm{n}=9$
$\mathrm{n}=2$

\begin{tabular}{lccc}
\hline Beds & $21-74$ & $65-400$ & $525-540$ \\
Trauma-cases annually & $20-150$ & $25-350$ & $3500-5500$ \\
Doctors & $3-6$ & $5-13$ & $80->100$ \\
Specialists & $0(-2)$ & $0-4$ & $35-50$ \\
Surgeons & 0 & $0-1$ & $6-10$ \\
Nurse anaesthetist & $0-3$ & $2-6$ & $4-7$ \\
Physician anaesthetist & 0 & $0-1$ & $6-7$ \\
Doctors A\&E & 0 & 0 & $4-6$ \\
& & & \\
\hline
\end{tabular}


Table 2

Physical resources for initial resuscitation and diagnosis of trauma victims at the three hospital levels in Botswana.

Items designated as desirable in the guidelines are upgraded to essential because of Botswana's status as a middle-income country. Primary hospitals compares to GP-staffed hospitals in the resource matrix, district hospital to specialist hospital, and referral hospital to tertiary hospital.

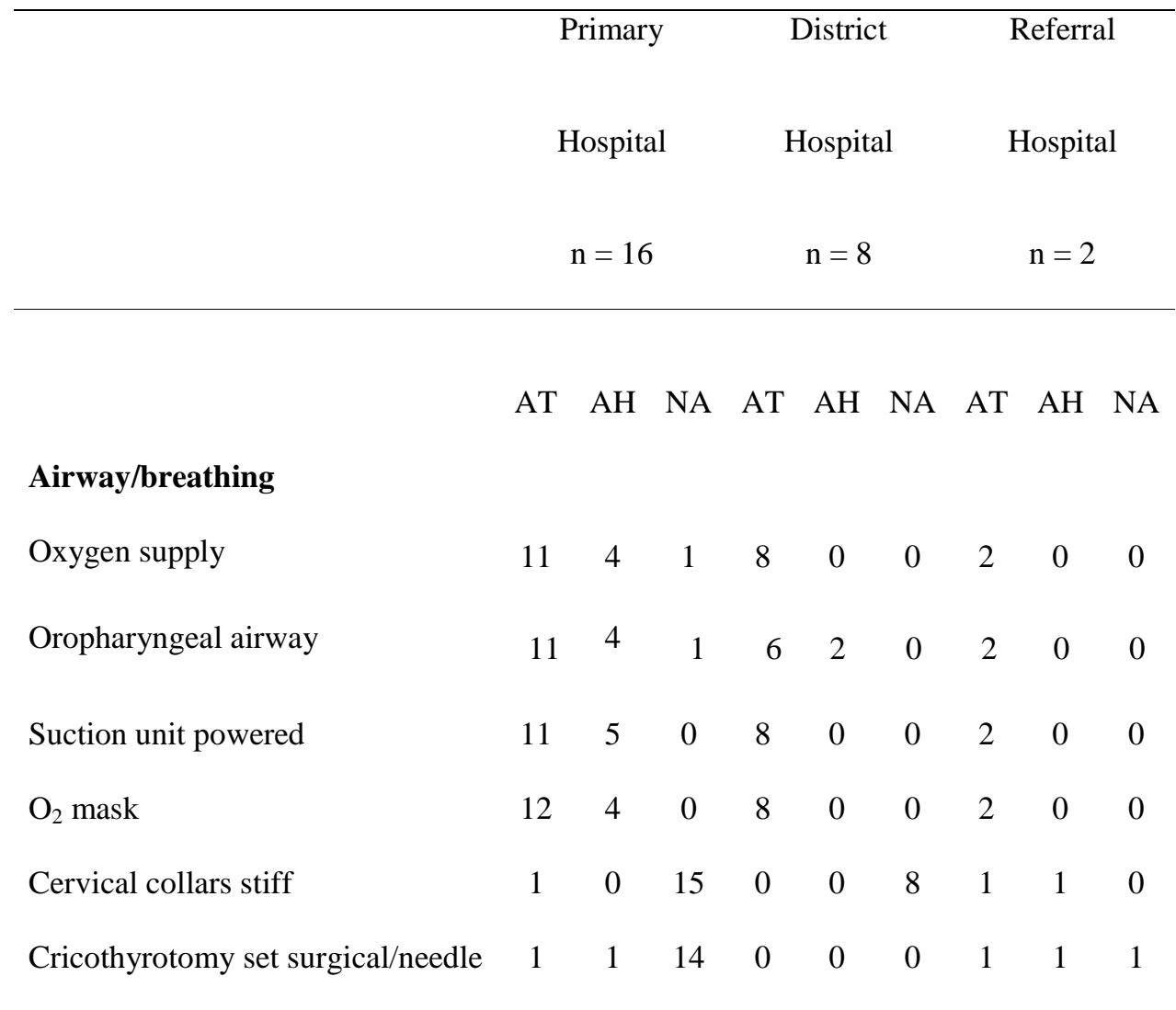




\begin{tabular}{|c|c|c|c|c|c|c|c|c|c|}
\hline Bag-valve-mask adult & 7 & 4 & 5 & 5 & 2 & 1 & 2 & 0 & 0 \\
\hline Bag-valve-mask paediatric & 4 & 5 & 7 & 5 & 2 & 1 & 1 & 0 & 1 \\
\hline Laryngoscope adult & 10 & 5 & 1 & 5 & 3 & 0 & 2 & 0 & 0 \\
\hline Laryngoscope paediatric & 9 & 4 & 2 & 5 & 3 & 0 & 2 & 0 & 0 \\
\hline Endotracheal tube adult & 7 & 7 & 2 & 6 & 2 & 0 & 2 & 0 & 0 \\
\hline Endotracheal tube (paediatric) & 6 & 7 & 3 & 6 & 2 & 0 & 2 & 0 & 0 \\
\hline Chest tubes (adult) & 6 & 8 & 2 & 4 & 4 & 0 & 2 & 0 & 0 \\
\hline Chest tubes (paediatric) & 3 & 4 & 9 & 3 & 3 & 2 & 2 & 0 & 0 \\
\hline NG tubes & 13 & 3 & 0 & 6 & 2 & 0 & 2 & 0 & 0 \\
\hline Pulse oximeter & 1 & 11 & 4 & 4 & 4 & 0 & 1 & 1 & 0 \\
\hline Arterial blood gas & 0 & 0 & 16 & 0 & 0 & 8 & 0 & 2 & 0 \\
\hline Ventilator & - & - & - & 1 & 7 & 0 & 2 & 0 & 0 \\
\hline \multicolumn{10}{|l|}{ Circulation } \\
\hline Crystalloids & 15 & 1 & 0 & 8 & 0 & 0 & 2 & 0 & 0 \\
\hline Colloids & 10 & 4 & 2 & 5 & 2 & 1 & 2 & 0 & 0 \\
\hline IV access/infusion & 15 & 1 & 0 & 8 & 0 & 0 & 2 & 0 & 0 \\
\hline Intraosseus needle & 0 & 0 & 16 & 1 & 1 & 6 & 0 & 1 & 1 \\
\hline Fluid warmer & 1 & 5 & 10 & 0 & 2 & 6 & 0 & 1 & 1 \\
\hline Blood transfusion capabilities & 16 & 0 & 0 & 8 & 0 & 0 & 2 & 0 & 0 \\
\hline ECG & 1 & 8 & 7 & 2 & 2 & 4 & 1 & 1 & 0 \\
\hline Hb/electrolytes & 16 & 0 & 0 & 8 & 0 & 0 & 2 & 0 & 0 \\
\hline
\end{tabular}




\begin{tabular}{lccccccccc}
\hline Urinary catheters & 15 & 1 & 0 & 8 & 0 & 8 & 2 & 0 & 0 \\
Surgical sets venous cutdown & 3 & 2 & 11 & 3 & 0 & 5 & 1 & 0 & 1
\end{tabular}

\section{Diagnosis/other}

\begin{tabular}{lccccccccc} 
Plain radiological films & 1 & 14 & 1 & 1 & 7 & 0 & 0 & 2 & 0 \\
Portable films & 0 & 4 & 12 & 1 & 3 & 4 & 0 & 2 & 0 \\
Ultrasound & 0 & 2 & 14 & 0 & 5 & 3 & 0 & 2 & 0 \\
CT & - & - & - & 0 & 0 & 8 & 0 & 2 & 0 \\
Splint for fracture immobilization & 0 & 3 & 10 & 2 & 2 & 4 & 1 & 1 & 0 \\
\hline Emergency laparotomy sets & 0 & 13 & 3 & 0 & 8 & 0 & 0 & 2 & 0 \\
& & & & & & & & &
\end{tabular}

IV = intravenous; ECG=electrocardiogram; $\mathrm{Hb}=$ haemoglobin; $\mathrm{CT}=$ computed tomography;

AT = available in emergency room; $\mathrm{AH}=$ available in hospital; NA = not available; - = not applicable to that level

Data is missing for one district hospital. 
Table 3.

Knowledge, skills and human resources in Botswana trauma hospitals assessed in the format of questioning, "How often will your hospital be able to perform the following procedure?” stratified into $\mathrm{N}=$ never, $\mathrm{S}=$ sometimes, and $\mathrm{A}=$ always $(24 / 7 / 365) .-$ = not applicable to that hospital level.

Desireable resources in the guidelines are upgraded to essential because of Botswana position as middle income country. Primary hospital corresponds to GP, district to specialist and referral to tertiary hospital in the resource matrix.

Primary Hospital strict Hospital Referral Hospital

$$
\mathrm{n}=16 \quad \mathrm{n}=8 \quad \mathrm{n}=2
$$

$\begin{array}{llllllllll}\text { A } & \mathrm{S} & \mathrm{N} & \mathrm{A} & \mathrm{S} & \mathrm{N} & \mathrm{A} & \mathrm{S} & \mathrm{N}\end{array}$

\section{Airway/breathing}

\begin{tabular}{lccccccccc} 
Adm of $\mathrm{O}_{2}$ & 14 & 2 & 0 & 7 & 1 & 0 & 2 & 0 & 0 \\
Chin lift/jaw thrust & 3 & 13 & 0 & 3 & 5 & 0 & 2 & 0 & 0 \\
Insertion of oropharyngeal airway & 1 & 15 & 0 & 4 & 4 & 0 & 2 & 0 & 0 \\
Recovery position & 2 & 7 & 7 & 1 & 3 & 4 & 2 & 0 & 0 \\
\hline
\end{tabular}




\begin{tabular}{lccccccccc}
\hline Log roll & 0 & 5 & 11 & 0 & 3 & 5 & 2 & 0 & 0 \\
Asssisted ventilation & 2 & 13 & 1 & 4 & 4 & 0 & 2 & 0 & 0 \\
Endotracheal intubation & 0 & 13 & 3 & 3 & 5 & 0 & 2 & 0 & 0 \\
Needle thoracostomy & 0 & 10 & 6 & 0 & 6 & 2 & 1 & 1 & 0 \\
Chest tube insertion & 6 & 10 & 0 & 3 & 5 & 0 & 2 & 0 & 0
\end{tabular}

\section{Circulation}

Assessment shock

Monitoring

IV access

Intraosseus access

Peripheral cutdown

Deep packing

Pelvic wrap

Transfusion capabilities

Head injuries

Consciousness, pupils

Monitor/treat ICP

CT

Burr holes

$\begin{array}{ccccccccc}3 & 13 & 0 & 2 & 6 & 0 & 2 & 0 & 0 \\ 4 & 8 & 4 & 2 & 6 & 0 & 2 & 0 & 0 \\ 14 & 2 & 0 & 8 & 0 & 0 & 2 & 0 & 0 \\ 0 & 0 & 16 & 0 & 1 & 7 & 1 & 1 & 0 \\ 1 & 12 & 3 & 0 & 6 & 2 & 1 & 1 & 0 \\ 0 & 4 & 12 & 0 & 3 & 5 & 1 & 1 & 0 \\ 0 & 3 & 13 & 0 & 2 & 6 & 1 & 1 & 0 \\ 14 & 2 & 0 & 7 & 1 & 0 & 2 & 0 & 0\end{array}$

$$
\begin{array}{ccccccccc}
5 & 10 & 1 & 4 & 4 & 0 & 2 & 0 & 0 \\
- & - & - & - & - & - & 0 & 2 & 0 \\
- & - & - & 0 & 0 & 8 & 2 & 0 & 0 \\
- & - & - & 0 & 0 & 8 & 1 & 1 & 0
\end{array}
$$




\section{Neck injuries}

Surgical skills for

exploration

\section{Chest injuries}

Skills/equipment thoracotomy

$$
\begin{array}{llllll}
0 & 0 & 8 & 1 & 1 & 0
\end{array}
$$

\section{Abdominal injuries}

$\begin{array}{lccccccccr}\text { DPL } & 0 & 1 & 15 & 0 & 3 & 5 & 1 & 1 & 0 \\ \text { Ultrasonography } & 0 & 2 & 14 & 4 & 3 & 1 & 2 & 0 & 0 \\ \text { Skills/equipment laparotomy } & 2 & 6 & 8 & 1 & 5 & 2 & 2 & 0 & 0\end{array}$

\section{Extremities}

$\begin{array}{lccccccccr}\text { Basic immobilization (sling, splint) } & 9 & 4 & 3 & 5 & 3 & 0 & 2 & 0 & 0 \\ \text { Closed reduction fractures } & 12 & 4 & 0 & 5 & 3 & 0 & 2 & 0 & 0 \\ \text { Skin traction in fractures } & - & - & - & 2 & 5 & 1 & 2 & 0 & 0 \\ \text { Skeletal traction in fractures } & - & - & - & 0 & 1 & 7 & 1 & 1 & 0 \\ \text { External fixation } & - & - & - & 0 & 0 & 8 & 2 & 0 & 0 \\ \text { Internal fixation } & - & - & - & 0 & 0 & 8 & 1 & 1 & 0\end{array}$


Fasciotomy CS

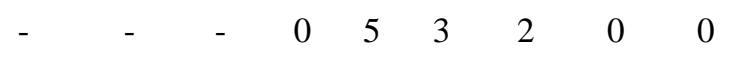

Amputation $\quad-\quad-\quad-\quad \begin{array}{lllllllll} & 0 & 3 & 5 & 2 & 0 & 0\end{array}$

IV = intravenous; CT = computed tomography; ICP = intracranial pressure; DPL = diagnostic peritoneal lavage; CS = compartment syndrome

Data is missing for one district hospital. 
- 33 - 